\title{
Research on RSSI-Based Localization System in the Wireless Sensor Network
}

\author{
Zhang Honghui* and Guo Rongyan
}

\begin{abstract}
School of Physics and Mechanical \& Electrical Engineering, Zhoukou Normal University, Zhoukou, Henan, 466001, China
\end{abstract}

\begin{abstract}
Due to the rapid development in wireless sensor networks, the applications of the WSN have been extended to different environment and achieved a more diversified purpose. AS for the localization system, there are many related research achievements. A set of localization system is built through the system integration between the small-scale wireless hardware sensor and the wireless sensor platform, through the use of the RSSI and the integration of the fuzzy inference system. The blind nodes are experimented in the indoor environment so that the system's efficiency can be verified. The system is implemented in the 8 meters multiplying 8 meters indoor space which is divided into 16 sections. Then 4 anchor nodes are placed in the corners and the fuzzy identification system is built for the nodes' RSSI signal in the blocks. Later, the localization test is implemented in the random positions within the blocks. The experimental results show that the localization system accuracy rate is more than $80 \%$ and can successfully reach the preliminary localization system.
\end{abstract}

Keywords: Fuzzy inference system, localization, RSSI, wireless sensor network.

\section{INTRODUCTION}

With the rapid development in wireless sensor networks, the applications of the WSN is more diversified such as in the intelligent residential areas, the environmental monitoring, the human body monitoring, the ecological monitoring, the mudslide monitoring, the military defense, the health \& medical and other fields. The measured parameters in the environmental monitoring include temperature, humidity, gas concentration and other data. These data can be regarded as the judgment criterion to the development of forests and agriculture. The data in the human body monitoring include heartbeat, blood pressure, body temperature and others. These data can be used as the medical judgment criterion and monitor human's physical condition [1].

The position of the wireless sensor node in the above applications becomes an important issue. Most of the transferred information packets just include the required monitoring data without its position of the wireless sensor node. When some sensor nodes have the mobile function, the localization of the sensor node is an important research topic.

The outdoor localization research is very widespread and sophisticated with the use of the Global Position System. GPS adopts the satellite to obtain GPS devices' longitude\&latitude coordinates, speed, time and other related information, but its disadvantage is that it has high costs and can not receive the indoors' correct satellite signals. Therefore, the indoor localization system with low costs and low electricity consumption should be emphasized and its achievements should be applied in the indoor precious experimental equipment's tracking localization research $[2,3]$.

There are many common localization technologies in the localization aspect. For example, the Angle of Arrival adopts the angles of receiving signals to implement its localization, but it requires extra directive antennas to finish its localization. The Time of Arrival and Time Difference of Arrival adopts the time of receiving signals to implement its localization, but its synchronization part of the signals time among nodes require extra hard wares to finish its localization.The Received Signal Strength Indicator adopts the RSSI to implement its localization without extra and high-cost hard wares, and all RF chips can provide the digitized RSSI parameters. Therefore, the RSSI-based localization which is convenient and simple is used as the target in the paper.

The localization is generally researched with the use of the RSSI and the Link Quality Indicator. Many related researches convert the signal strength into the distance and then the localization system is finished with the use of the algorithms. When the formula in which the RSSI values are converted into distances is obtained, its localization error is at least above 2 meters. Therefore, when the RSSI values are converted into distances, the related parameters should be set up and its parameter setting can affect its localization accuracy. A mass of localization researches just can be shown in the computer simulational results. As the RSSI can be influenced by the signal reflection, the diffraction, the shelter and others, the influenced values can cause more errors during the process of converting the RSSI values into distances. Hence, The localization researches will be done with the relative relationship of the RSSI signals $[4,5]$. 

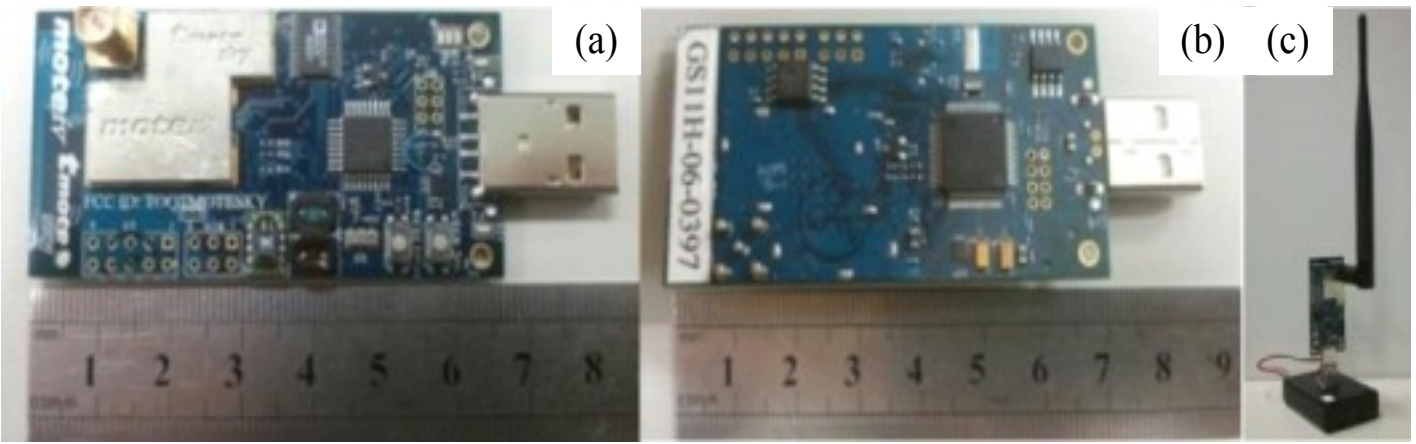

Fig. (1). (a) is the front side of the mote, (b) is the back side of the mote and (c) is equipment integration figure.

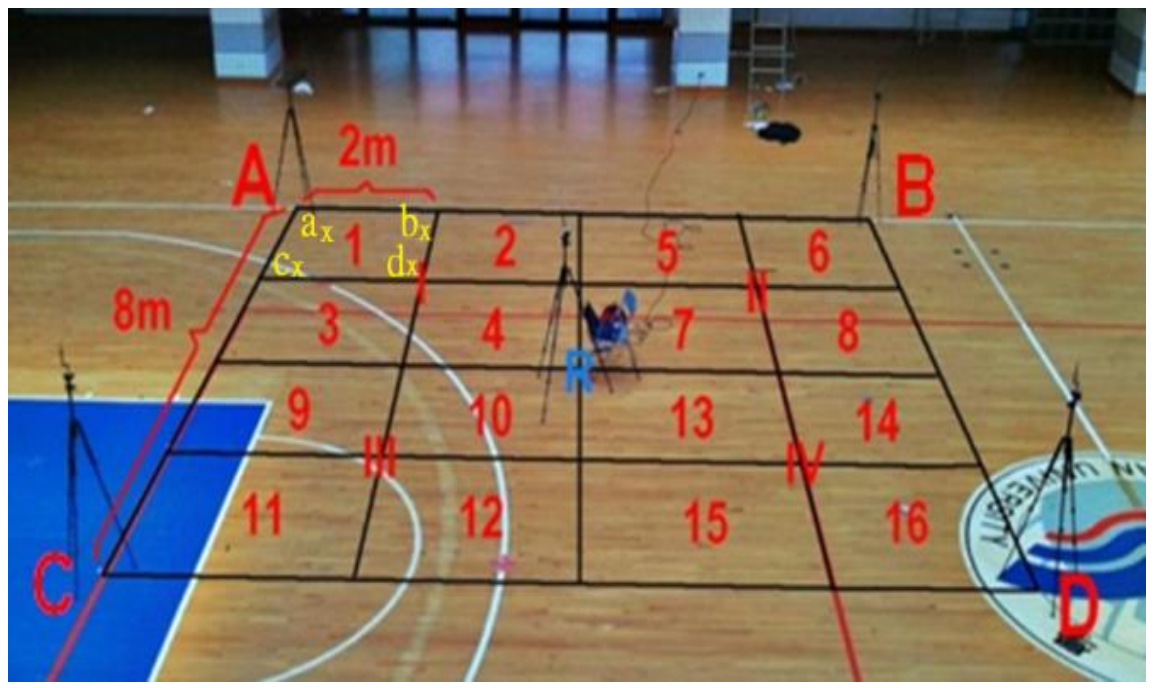

Fig. (2). Actual experimental site.

\section{HARD WARES INTRODUCTION}

The basic hardware mote adopts the Tmote Sky module (as shown in the Fig. (1a) is the front side and (1) is the back side.), the software developing interface is the TinyOS 2.0.5 version' embedded operating system of the open source, the programming language is nesC and the integration of the back-end data presents its results by writing MATLAB program. The wireless sensor node is the Tmote Sky made by the Moteiv, including TI MSP430 processor, CC2420 RF chip and $\mathrm{F}$ type antenna. Its module communicating range is that its indoor is 50 meters and its outdoor is 125 meter [6]. TI MSP430 processor has many advantages, such as many kinds of power-saving modes, the function of being waking up quickly $(<6 \mu \mathrm{s})$, the FLASH internal memory with the $48 \mathrm{~KB}+256 \mathrm{~KB}$, many simulations, digital input\&output connection tubs and others. The CC2420 RF chip adopts the wireless RF chip based on the ZigBee protocol of the IEEE 802.15.4 and has the low power consumption and low voltage wireless application. The CC2420 RF chip can also offer the RSSI buffer and their values can be read directly and its output power is controlled by the Transmit Control Register. The experiment sets it as the $0 \mathrm{dBm}$ which is the maximum output power [7].

The F type antenna within the Tmote Sky should be built, but its display ways, no matter which is up-right, flat-wise or lean, can affect the RSSI to a certain degree so that it should be based on a more accurate received signals. In addition, the $5 \mathrm{dBi}$ omni-directional antenna should be installed in the nodes, its antennas' displaying angles should be vertical to the ground and the antenna's frequency range is from 2.405 $\mathrm{Hz}$ to $2.484 \mathrm{GHz}$. The experiment is tested by the environment and the $2.48 \mathrm{GHz}$ whose noise interference is smaller should be selected. The Fig. (1c) is the equipment integration figure of Tmote sky, the antenna and the battery box. The battery box adopts three $1.5 \mathrm{~V}$ alkaline batteries to supply power.

\section{RESEARCH METHODS}

The square whose space is 8 meters multiplying 8 meters is the actual experimental site (as shown in the Fig. 2), which is the gymnasium where there is an indoor, no shelter above and no surrounds all around. The site is divided into 16 blocks whose space is 2 meters multiplying 2 meters, and the numbers in the blocks represent the block positions. The site's up, down, left and right of the black frame lines in the Fig. (2) is respectively represented by A, B, C and D. All of them is respectively the 4 nodes of the transmitting terminal and placed in the corner of the square whose space is 8 meters multiplying 8 meters. $\mathrm{R}$ is the receiving terminal node [8, 9]. The building of the experimental system model is to 


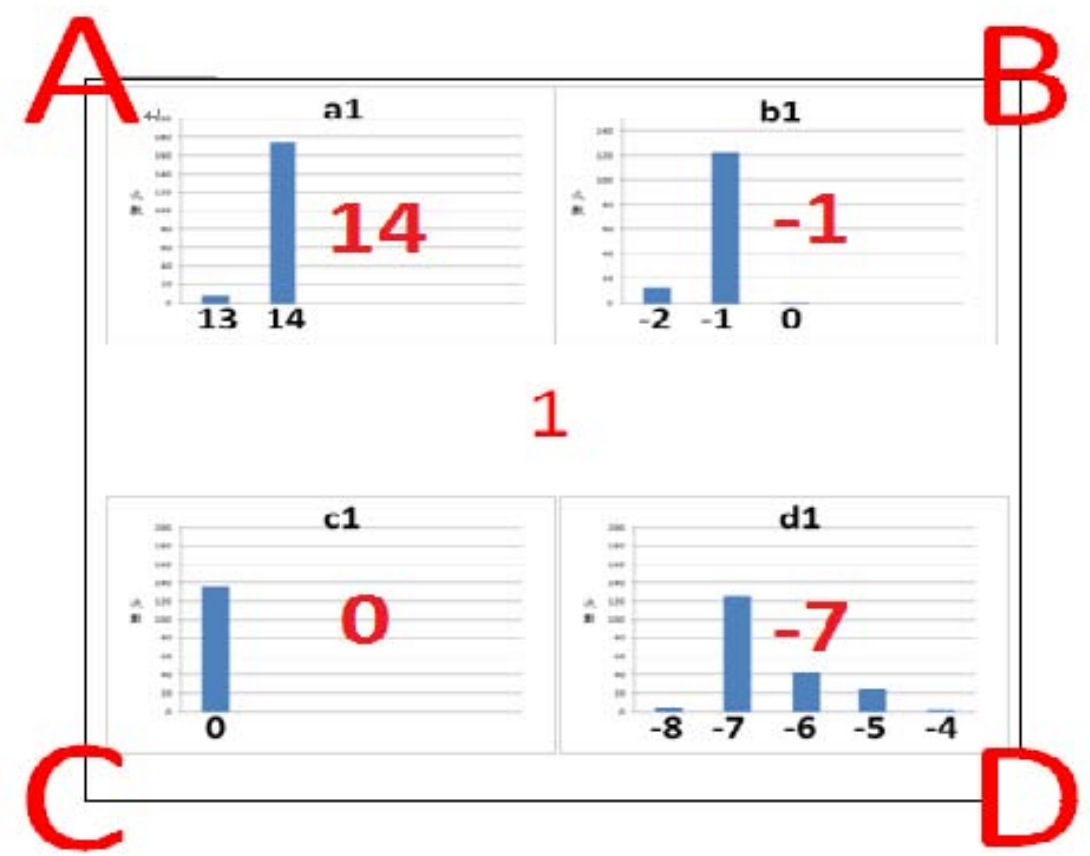

Fig. (3). The experimental data distribution figure in the block 1 .

place the receiving terminal $\mathrm{R}$ in the center of each block (means the red position in each block). The nodes between the transmitting terminals and the receiving terminals should be installed the tripods and it can be experimented when those nodes are about 1.5 meters from the ground.

The transmitting terminal A, B, C and D can simultaneously transmit the signals with the broadcasting method during the process of the experiments. The receiving $\mathrm{R}$ can receive the signals derived from the 4 groups of A, B, C and D each second. The CC2420 RF chip can offer the RSSI buffer and the data of the RSSI can be directly read. The experimental time in each block is one minute, and the total data obtained from the single transmitting terminal's nodes is 240 . The 240 experimental data adopt the mode method to determine its RSSI valued obtained from the experiment. The Fig. (3) is the RSSI data distribution figure received from from the transmitting terminal $\mathrm{A}, \mathrm{B}, \mathrm{C}$ and $\mathrm{D}$ in the block 1 . The RSSI value received from the A, B, C and D signal with the selection of the mode method is respectively $14,-1,0$ and -7 . The RSSI values is not only influenced by the shelters, surroundings, but also the temperature and humidity. The experiment uses the data which is continuously measured for a long time as the referent criterion [10].

The RSSI received from the $\mathrm{A}, \mathrm{B}, \mathrm{C}$ and $\mathrm{D}$ signal is marked as ax, bx, cx and $\mathrm{dx}$, in which $\mathrm{x}$ means the block marks from 1 to 16 , that is, the RSSI data received from the $\mathrm{A}, \mathrm{B}, \mathrm{C}$ and D signal in the block $\mathrm{x}$. For example, when the $\mathrm{a} 1, \mathrm{~b} 1, \mathrm{c} 1$ and $\mathrm{d} 1$ is in the block 1 , the RSSI data can be received from the $\mathrm{A}, \mathrm{B}, \mathrm{C}$ and $\mathrm{D}$ signal.

\subsection{Signal Strength Analysis}

The RSSI buffer offered by the CC2420 can be reverted to the actual $\mathrm{dBm}$ value through computation. The value in the RSSI buffer is represented by RSSI_VAL and the RSSI_OFFSET is the gaining experience values derived from the front side during the development of the system [11, 12]. The approximating value of the RSSI_OFFSET is -45 . For example, the value read in the RSSI buffer is -20 and the RF's output power is about $-65 \mathrm{dBm}$.

$$
P=R S S I_{-} V A L+R S S U U_{-} \text {OFFSET } \quad[\mathrm{dBm}]
$$

As the wave radiation strength and the square of the distance have an inverse relationship, the distance between the transmitting terminal nodes and the receiving terminal node should be discussed. The Fig. (4) is the distance between the node $\mathrm{A}$ and each receiving terminal. The same color block represents the same distance and the signal strength is represented by the class $\mathrm{S}$. The 16 blocks are divided by the class and then should be integrated into the Table 1. T represents the distance between the transmitting terminal nodes and the receiving terminal nodes. The experiment's signal strength range lies in the right of the segments.

\subsection{The Fuzzy Inference System}

The extracted RSSI data is not the single data but a numeric range. If the extracted data is judged by the threshold value dichotomy, it can easily produce errors. Therefore, the localization system in the block adopts to the fuzzy inference system.

\subsubsection{Input And Output Parameters}

The input and output parameters should be set up in the fuzzy inference system in terms of many experimental data and then the number and shape of the membership function should be defined. The input parameters in the system mean the 4 RSSI values received by the receiving terminal 


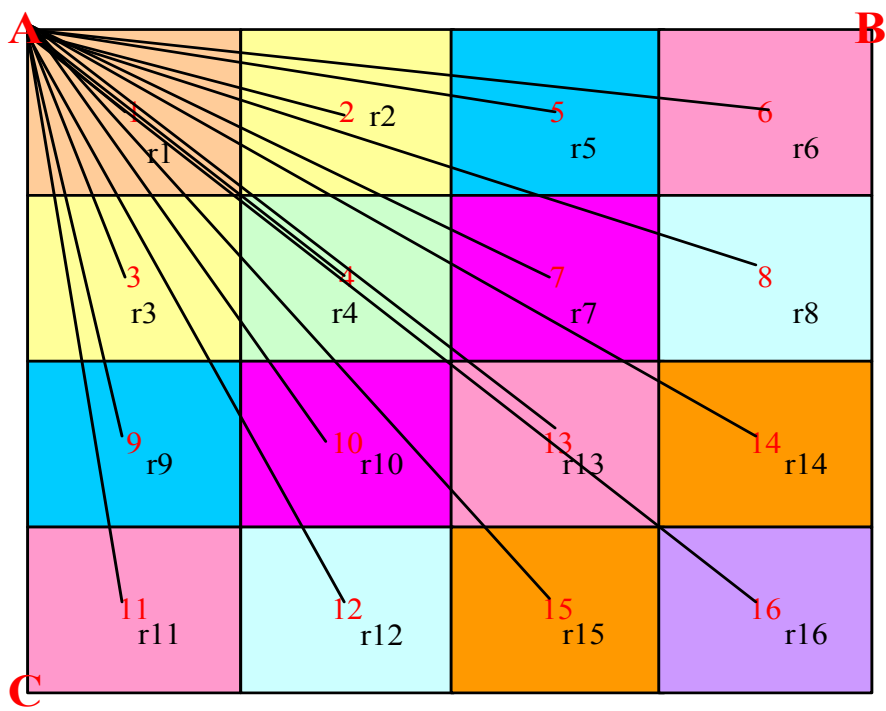

Fig. (4). The wave radiation figure in the center of A.

Table 1. The Actual Distance Between The Node A And Each Block And The RSSI Integration Table.

\begin{tabular}{|c|c|c|c|}
\hline Class & Block Number & Actual Distance & RSSI Signal \\
\hline \hline S1 & 1 & $\mathrm{r} 1$ & $10 \sim 18$ \\
\hline S2 & $2,3, \mathrm{r} 3$ & $2 \sim 9$ \\
\hline S3 & 4 & $\mathrm{r} 4$ & $2 \sim 4$ \\
\hline S4 & 5,9 & $\mathrm{r} 5, \mathrm{r} 9$ & $-1 \sim 1$ \\
\hline S5 & 7,10 & $\mathrm{r} 7, \mathrm{r} 10$ & $-3 \sim 1$ \\
\hline S6 & $6,11,13$ & $\mathrm{r} 8, \mathrm{r} 12$ & $-\mathrm{r} 13$ \\
\hline S7 & 8,12 & $\mathrm{r} 14, \mathrm{r} 15$ & $-4 \sim-1$ \\
\hline S8 & 14,15 & $\mathrm{r} 16$ & $-8 \sim-2$ \\
\hline S9 & 16 & $-13 \sim 0$ \\
\hline
\end{tabular}

experiments one minute, respectively are ax, bx, cx and $\mathrm{dx}$. The ideal output parameters is from the block 1 to the block 16 , the membership function defined by the system has 4 types: L1, L2, L3 and L4 and its shape is the trapezoidal, as shown in the Fig. (5).

\subsubsection{The Building Of The Membership Function}

Its experimental environment is the 16 blocks' positions and the membership function can be drawn up according to each antenna's wave radiation, actual distance and RSSI data, as shown in the Fig. (4). The transmitting $A$ is the center of the wave radiation figure, and the same color blocks represent the distances between the block and the transmitting A is the same. Each transmitting terminal is the center and the experimental environment can be divided into 9 kinds of classes, the Table 1 takes the transmitting A as an example. The RSSI data range received from the blocks which are far away from the center point is so float that the 9 kinds of classes can be merged into 4 kinds of classes to bui8ld the membership function. Many experimental results show that the RSSI membership function can be represented by the trapezoidal distribution, and its relative values can be shown from the formula (1) to the formula (4), as shown in the Fig. (5).

(1) L1

$L 1(x)=\{0|x \leq 9, x-9| 9<x<10,1 \mid x \geq 10\}$

(2) L2

$L 2(x)=\left\{\begin{array}{c}0|x \leq 5, x-5| 5<x<6, \\ 1|6 \leq x \leq 9,10-x| 9<x<10, \\ 0 \mid x \geq 10\end{array}\right\}$ 


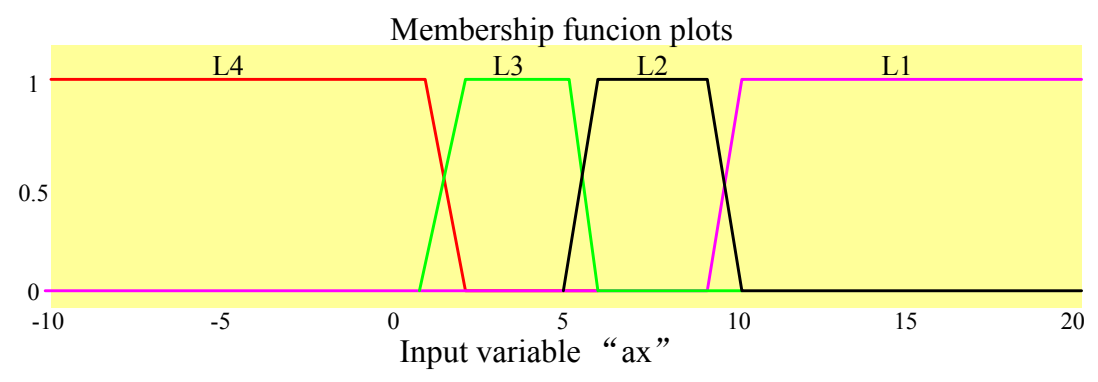

Fig. (5). The membership function of the RSSI.

Table 2. The Fuzzy Rule Base.

\begin{tabular}{|c|c|c|c|c|c|c|c|c|c|}
\hline Block & $\mathbf{a}_{\mathbf{x}}$ & $\mathbf{b}_{\mathbf{x}}$ & $\mathbf{c}_{\mathrm{x}}$ & $d_{x}$ & Block & $\mathbf{a x}$ & $\mathbf{b}_{\mathbf{x}}$ & $\mathbf{c}_{\mathrm{x}}$ & $d_{x}$ \\
\hline 1 & $\mathrm{~L} 1$ & L4 & L4 & L4 & 9 & L3 & L4 & L2 & L4 \\
\hline 3 & L2 & L4 & L3 & L4 & 11 & L4 & L4 & L1 & L4 \\
\hline 5 & L3 & L2 & L4 & L4 & 13 & L4 & L4 & L4 & L3 \\
\hline 6 & L4 & L1 & L4 & L4 & 14 & L4 & L3 & L4 & L2 \\
\hline 7 & $\mathrm{~L} 4$ & L3 & L4 & L4 & 15 & L4 & L4 & L3 & L2 \\
\hline
\end{tabular}

(3) L3

$$
L 3(x)=\left\{\begin{array}{c}
0|x \leq 1, x-1| 1<x<2, \\
1|2 \leq x \leq 5,6-x| 5<x<6, \\
0 \mid x \geq 6
\end{array}\right\}
$$

(4) L4

$$
L 4(x)=\{1|x \leq 1,2-x| 1<x<2,0 \mid x \geq 2\}
$$

\subsubsection{The Building of the Fuzzy Rule Base}

The fuzzy rule base adopts the fuzzy rule which is if... and...then... To describe the input and output relationship of the system, as shown in the Table 2, such as if (ax is L1) and (bx is L4) and (cx is L4) and (dx is L4) then (the block is " 1 "). The block positions can be localized with the use of the rule base. The rule base has 16 kinds of combinations for there are 16 block positions.

\section{RESEARCH METHODS}

The built fuzzy inference should be carried on the actual blind node test to verify the effect of the research. When the blind nodes are tested, the sensor nodes should be placed, and then receive the RSSI values from the transmitting A, B, $\mathrm{C}$ and D. After being experimented one minute, the RSSI data can be obtained with the mode method. Later, the obtained experimental data can be adopted to identify the blind nodes and verify whether the block positions are right or not through the built fuzzy inference system.

The blind node experiment can be divided into two types: the blind node experiment which is in the center of the blocks (as shown in the Fig. 6) and the blind node experiment whose deviation distance is about $50 \mathrm{~cm}$ from the center of the blocks (as shown in the Fig. 7). The two kinds of the blind node experiments are tested 50 times respectively and the identified accuracy of the blind nodes is as shown in the Table 3. The blind nodes of the wrong localization is marked by the blue lines. After being tested 50 times to the blind nodes, the accuracy of the blind node experiments in the center of the blocks is $84 \%$, while the accuracy of the blind node experiment whose deviation distance is about 50 $\mathrm{cm}$ from the center of the blocks is $80 \%$.

There are two kinds of reasons to cause the error localization results. The first kind is that the changing values of the received RSSI data is larger and its range may stretch across two membership functions. Therefore, a membership function classification can not require its meet so that the extra situation except the rule base can be caused and then the wrong localization can be caused during the process of 


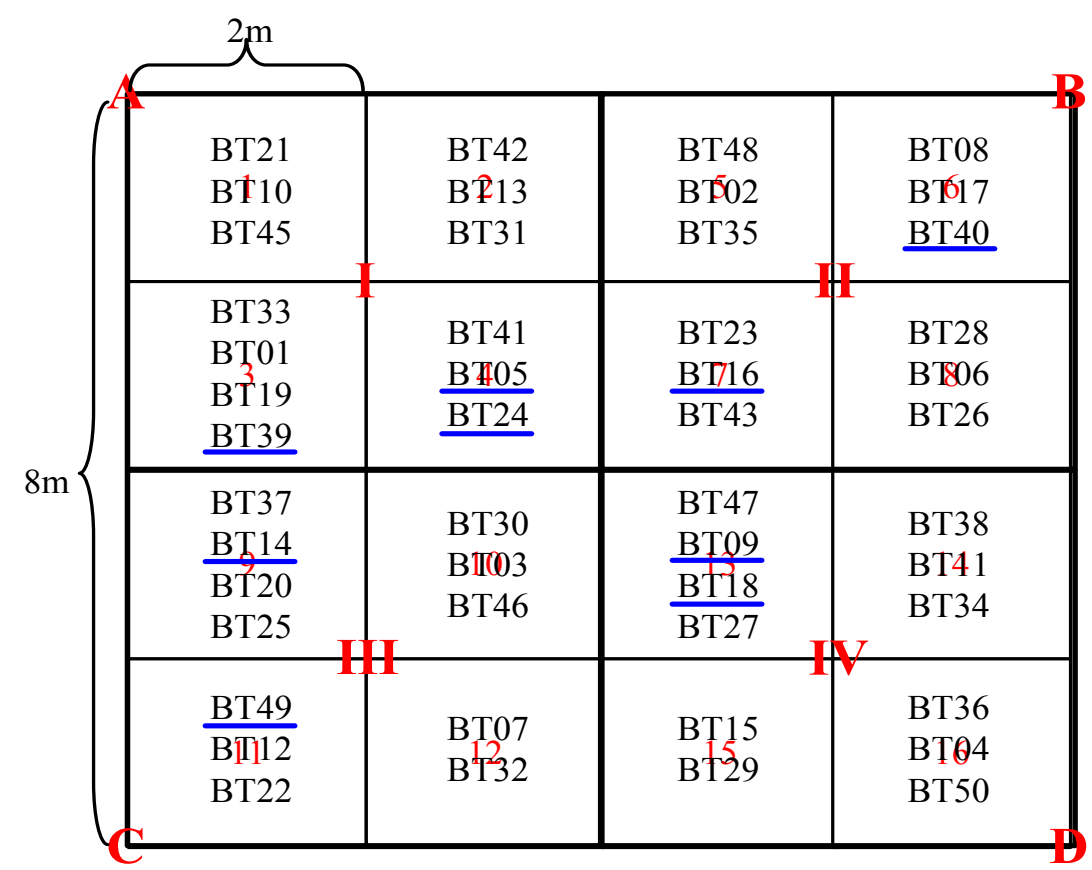

Fig. (6). The position of the blind nodes experiments in the center of the blocks.

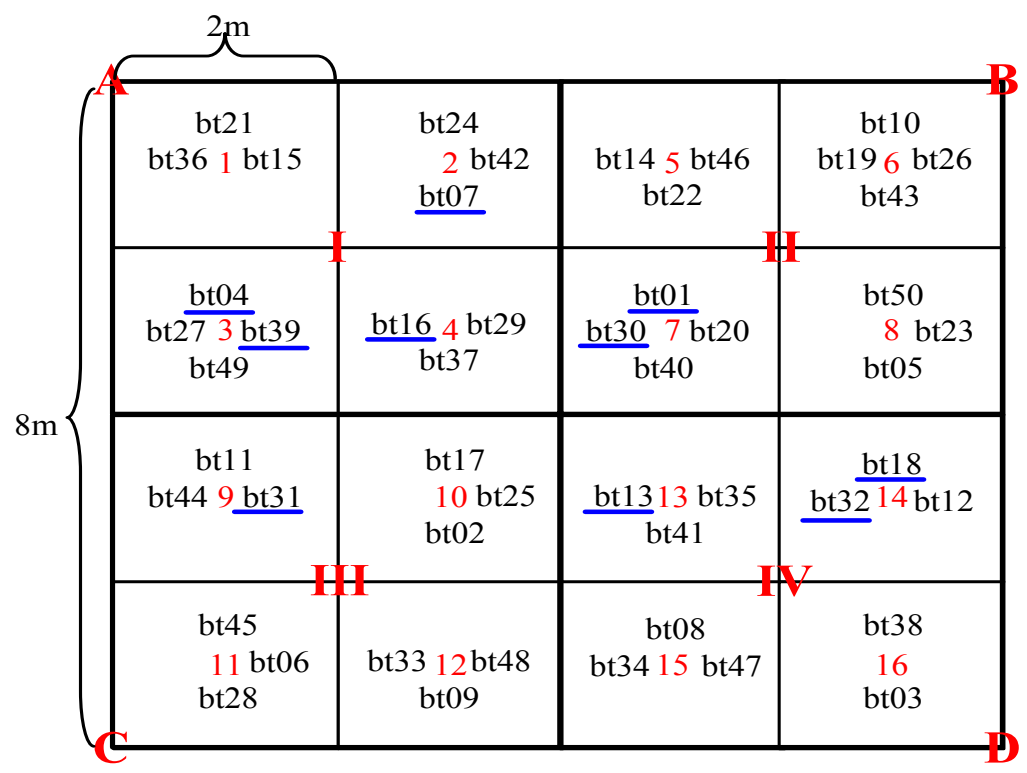

Fig. (7). The blind nodes experiment figure whose deviation distance is $50 \mathrm{~cm}$ from the center of the blocks.

Table 3. The Experimental Data Of The Blind Node Localization.

\begin{tabular}{|c|c|}
\hline The Experimental Position of the Blind Nodes & Accuracy \\
\hline \hline The center of the blocks & $84 \%$ \\
\hline The position whose deviation distance is $50 \mathrm{~cm}$ from the center of the blocks & $80 \%$ \\
\hline
\end{tabular}


Table 4. The Experimental Data Of The Blind Node In The Center Of The Blocks.

\begin{tabular}{|c|c|c|c|c|c|c|c|}
\hline \multirow{2}{*}{ Experiments } & \multicolumn{4}{|c|}{ RSSI Data } & \multirow{2}{*}{ Actual Blocks } & \multirow{2}{*}{ Estimated blocks } & \multirow{2}{*}{ Results } \\
\hline & $\mathbf{a x}$ & $\mathbf{b}_{\mathbf{x}}$ & $\mathbf{c}_{\mathrm{x}}$ & $d_{x}$ & & & \\
\hline ВT9 & 0 & 2 & 3 & 4 & 13 & - & Unidentified \\
\hline BT16 & 2 & 5 & 3 & 2 & 7 & - & Unidentified \\
\hline BT18 & -3 & 2 & 6 & 4 & 13 & 12 & Error blocks \\
\hline BT24 & 4 & 1 & -2 & 2 & 4 & - & Unidentified \\
\hline BT39 & 7 & 2 & 1 & -3 & 3 & 2 & Error blocks \\
\hline
\end{tabular}

Table 5. The Experimental Data Of The Blind Node Whose Deviation Distance Is $50 \mathrm{~cm}$ From The Center Of The Blocks.

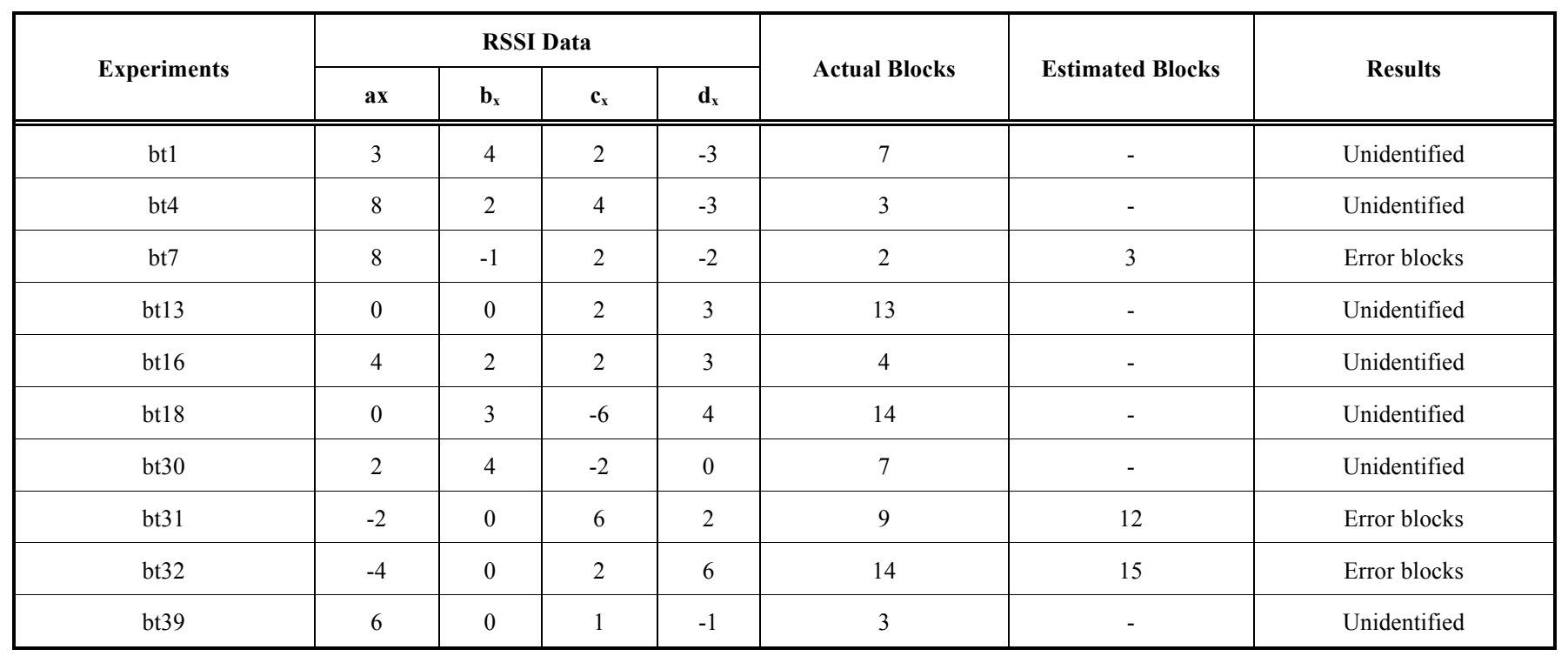

verifying the blind nodes. For example, the RSSI values in the experiment BT5 and BT49 changes so big that it can not be identified in the Table $\mathbf{4}$ whose blind node experiments is in the block position. The value bx, $\mathrm{dx}$ in the BT5 and the value $\mathrm{dx}$ in the BT49 are very big and not accord with the membership function condition of the original rule base so that the block positions can not be localized. The RSSI values in the Table 5 whose deviation distance is about $50 \mathrm{~cm}$ from the center of the blocks have the same situation. The value cx and the value ax in the BT13 are very big and the RSSI data is different from the fuzzy rule base so that those non-identified parameters are marked by the red lines.

The second kind is that it may be influenced by the antenna displaying angle problems of the nodes or the experimental environment during the process of the blind node experiment. The BT14, BT39 and BT14 in the Table 4 is placed in the block 9 to receive signal but data in the block 12 is obtained by the RSSI parameters. Therefore, the error problem many be caused by the experimental environment. The Table $\mathbf{5}$ is the blind node experiment which is deviated from the center of the blocks so that the BT7and BT32 have the same situation.

\section{CONCLUSION}

The RSSI signals can be transmitted into the computers with the wireless sensor nodes, and data can be localized to the receiving terminal nodes through the fuzzy inference system. As the RSSI values have a certain errors during the process of being converted into the distances, the relative relationship of the RSSI signals are used to do the localization research. After analyzing the efficiency of the localization 
systems with a mass of blind nodes, the experimental results show that the accuracy of the blind node experiments in the center of the blocks is $84 \%$, while the accuracy of the blind node experiment whose deviation distance is about $50 \mathrm{~cm}$ from the center of the blocks is $80 \%$.

With the changing of the RSSI parameters, a block with two or over two kinds of the fuzzy rules will be discussed in the future and the environmental parameters (such as temperature and humidity) will be increased to do the experimental tests with the bland nodes many times. The different parameters should be found out to be regarded as the input terminal and then added into the fuzzy system to improve the localization accuracy. The localization analysis with other algorithms will be compared with the achievements of the fuzzy localization system in the future.

\section{CONFLICT OF INTEREST}

The author confirms that this article content has no conflict of interest.

\section{REFERENCES}

[1] G. Mao, B. Fidan, and B.D.O. Anderson, "Wireless Sensor Network Localization Techniques," ACM Computer Networks, vol. 51, pp. 2529-2553, 2007.

[2] N. Patwari, A.O. Hero III, M. Perkins, N.S. Correal and R.J. O'Dea, "Relative Location Estimation in Wireless Sensor Networks," In: IEEE Trans. on Signal Processing, vol. 51, no. 8, pp. 2137-2148, 2003.
[3] Y. Wang, X. Xu, and X. Tao, "Localization in Wireless Sensor Networks via Support Vector Regression," In: Proceedings of International Conference on Genetic and Evolutionary Computing, pp. 549-552, 2009.

[4] P. Kumar and L. Reddy, "Distance measurement and error estimation scheme for RSSI based localization in Wireless ensor Networks," In: Proceedings of Wireless Communication and Sensor Networks, pp.1-4, 2009.

[5] X. Feng, Z. Gao, M. Yang, and S. Xiong, "Fuzzy Distance Measuring Based on RSSI in Wireless Sensor Network," In: Proceedings of International Conference on Intelligent System and Knowledge Engineering, pp. 395-400, 2008.

[6] J. Blumenthal, R. Grossmann, F. Golatowski, and D. Timmermann. "Weighted Centroid Localization in Zigbee-based Sensor Networks," In: Proceedings of IEEE International Symposium on Intelligent Signal Processing, 2007, pp.1-6.

[7] V. Daiya, J. Ebenezer, S.A.V.S. Murty, and B. Raj, "Experimental Analysis of RSSI for Distance and Position Estimation" In: Proceedings of IEEE-International Conference on Recent Trends in Information Technology, 2011, pp.1093-1098.

[8] Moteiv Corporaton, Tmote Sky: Datasheet, Available at http://www.bandwavetech.com/download/tmote-sky-datasheet.pdf.

[9] Texas Instruments Incorporated MSP430F15x, MSP430F16x, MSP430F161x mixed signal microcontroller: Datasheet Available at http://www.alldatasheet.com/datasheet- pdf/pdf/27250/TI/MSP430.html.

[10] Chipcon CC2420: Data sheet available at http://www.ti.com/lit/ds/symlink/cc2420.pdf

[11] S.Z. Erdogan, and S. Hussain, "Using Received Signal Strength Variation for Energy Efficient Data Dissemination in Wireless Sensor Networks", In: The 18th International Conference on Database and Expert Systems Applications, 2007, pp. 620-624.

[12] J. Yin, Q. Yang, and L. Ni, "Adaptive temporal radio maps for indoor location estimation," In: Proceedings of IEEE International Conference on Pervasive Computing and Communications, 2005, pp. 85-94.

(C) Honghui and Rongyan; Licensee Bentham Open.

This is an open access article licensed under the terms of the Creative Commons Attribution Non-Commercial License (http://creativecommons.org/licenses/by-nc/3.0/) which permits unrestricted, non-commercial use, distribution and reproduction in any medium, provided the work is properly cited. 